\title{
EDUCAÇÃO EM SAÚDE NA FORMAÇÃO DE DISCENTES DE ENFERMAGEM
}

\author{
HEALTH EDUCATION IN THE FORMATION OF NURSING STUDENTS \\ Tatiana Almeida Couto, Rose Manuela Marta Santos, Sérgio Donha Yarid
}

Universidade Estadual do Sudoeste da Bahia - UESB

\begin{abstract}
The objective of this study is to conduct a survey in the national and international literature about the perspectives of nursing student training in the context of health education. It consists of na integrative review based on the search in the data bases of the Virtual Health Library Portal and the Scopus and Web of Science databases from Capes' Portal of Periodicals, using the descriptors "Health Education", "Students, Nursing" and "Education, Nursing" and combined with the boolean operator "AND". Original, fulltext studies published between 2012 and 2016 were documented to document the perspective of nursing student training in the context of health education, in the Portuguese, English and Spanish languages. The criterion of analysis o the topics that provided to categorize, elucidate and classify the data was applied. This grouping shows two categories: methodologies for training in health education and the studies found indicate that the experiences in extension projects, supervised internships, extracurricular internships, learning in virtual environment; conception of nursing students about health education in training, in which there is a predominance of the valorization of health education in the training and the need for motivation in the university for experiences in academic as well as social environments. It was observed that nursing education demands critical, flexible and active teaching.
\end{abstract}

Key words: Health Education; Education, Nursing; Students, Nursing; Education, Higher; Nursing.

\section{Resumo}

o objetivo deste estudo é realizar um levantamento na literatura nacional $e$ internacional acerca das perspectivas de formação do discente de enfermagem no contexto da educação em saúde. Consiste em uma revisão integrativa baseada na consulta nas bases de dados do Portal da Biblioteca Virtual em Saúde e nas bases de dados Scopus e Web of Science a partir do Portal de Periódicos da Capes, utilizando os descritores "Health Education", "Students, Nursing" e "Education, Nursing" $e$ combinados com o operador booleano "AND". Foram incluídos estudos originais, disponíveis na íntegra, publicados entre 2012 e 2016 que documentassem a perspectiva de formação do discente de enfermagem no contexto da educação em saúde, nos idiomas português, inglês e espanhol. Foi aplicado o critério de análise dos tópicos que proporcionou categorizar, elucidar e classificar os dados. Desse agrupamento mostra-se duas categorias: metodologias para a formação em educação em saúde e os estudos encontrados apontam que são oportunizadas as vivências em projetos de extensão, estágio supervisionado, estágio extracurricular, aprendizado em ambiente virtual; concepções de discentes de enfermagem sobre educação em saúde na formação na qual verificase a predominância da valorização da educação em saúde na formação e a necessidade de motivação na universidade para vivências em ambientes acadêmicos, assim como sociais. Observou-se que a formação em enfermagem demanda ensino crítico, flexível e ativo.

Palavras chave: Educação em Saúde; Educação em Enfermagem; Estudantes de Enfermagem; Educação Superior; Enfermagem. 


\section{Introdução}

A educação em saúde é uma prática social construída historicamente e permeada por subjetividades, intenções, objetos e fins. Assim, trata-se de um processo educativo que pode ou não envolver formalidade, sistematização ou intenção, que contribui para o desenvolvimento, humanização e inserção social dos indivíduos através da possibilidade de participação ativa, além da corresponsabilidade por sua saúde ${ }^{1}$.

A prática educativa a ser realizada pelo profissional de saúde pode colaborar para o usuário expressar suas necessidades de saúde, pois por meio das metodologias utilizadas por tais profissionais devem ser ressaltadas as questões sobre a autonomia do usuário, o empoderamento do indivíduo e da coletividade ${ }^{2,3}$.

A educação em saúde é considerada fundamental para suscitar que a participação dos usuários seja ativa e, assim, buscar a efetivação do Sistema Único de Saúde. Assim, os usuários e família empoderados poderão ter conhecimentos que motive para mudanças na prática quanto ao cuidado e terão suas percepções valorizadas, além de se tornarem potenciais multiplicadores de conhecimentos populares embasados também por conhecimentos científicos, através da aprendizagem significativa ${ }^{4,5}$.

A experiência demonstrada por usuários sobre o modo de conduzir a vida por meio de oportunidades vivenciadas em práticas educativas permite o conhecimento da dimensão subjetiva que essa atividade permite. Assim, nessa experiência entre profissional, usuário e família, deve ser valorizado o potencial de participação dos usuários e família a partir de sua realidade, que conduzirá à discussão de temáticas com conceitos e embasamento inatos à sua realidade social, econômica, política ${ }^{6}$.

No contexto da Estratégia de Saúde da Família (ESF), a educação em saúde para a população adscrita trata-se de uma das atribuições comuns a todos os profissionais da equipe da ESF e deve ser assegurada no planejamento e no desenvolvimento de tais atividades, a participação e interação dos usuários e da equipe ${ }^{7}$. Além disso, apesar das ações de promoção em saúde e prevenção de agravos serem fundamentalmente concebidas para serem articuladas na atenção primária, diante do número crescente de internações, percebe-se a necessidade da realização de ações de educação em saúde na assistência secundária e terciária ${ }^{8}$.

Portanto, diante das exigências na formação de discentes em Enfermagem, as Diretrizes
Curriculares Nacionais para o Curso de Graduação em Enfermagem evidenciam a necessidade de articulação entre os setores educação e saúde, para a efetivação de mudanças processuais na formação do enfermeiro com ênfase nos princípios e diretrizes do Sistema Único de Saúde (SUS) ${ }^{9}$. Considerando que a educação em saúde é uma atividade inerente a essa profissão.

Diante do exposto, emergiu a seguinte questão norteadora: quais as características da formação de discente de enfermagem em educação em saúde?

Assim, esse estudo tem como objetivo realizar um levantamento na literatura nacional e internacional acerca das perspectivas de formação do discente de enfermagem no contexto da educação em saúde.

\section{Metodologia}

Este trabalho consiste em uma revisão integrativa da literatura realizada em junho de 2017, baseada na consulta nas bases de dados do Portal da Biblioteca Virtual em Saúde (BVS) e nas bases de dados Scopus e Web of Science a partir do Portal de Periódicos da Capes. Os descritores em Ciências da Saúde (DeCS) utilizados foram "Health Education", "Students, Nursing" e "Education, Nursing" e combinados com o operador booleano "AND".

Os artigos identificados pela estratégia de busca foram avaliados inicialmente a partir da leitura dos títulos e resumos, e posteriormente a partir da leitura do artigo na íntegra, utilizando como critérios de inclusão nesta revisão: estudos originais, publicados entre 2012 e 2016, com texto completo disponível, que documentassem a perspectiva de formação de discentes de enfermagem no contexto da educação em saúde, nos idiomas português, inglês e espanhol. Foram excluídos artigos de reflexão, de revisão sistemática, integrativa e bibliográfica.

\section{Resultados e Discussões}

Após as buscas realizadas foram encontrados 168 artigos. Após a leitura de títulos e resumos e a supressão das produções duplicadas foram selecionadas 10 referências para leitura na íntegra.

A leitura das referências na íntegra e a análise interpretativa possibilitaram evidenciar o corpus do estudo ${ }^{10}$. 
Foi compilado as informações dos artigos, para facilitar a análise, a partir da organização e exposição dos resultados, destacando-se: os autores dos artigos; ano de publicação; objetivo da pesquisa; tipo de estudo e revista de publicação (Tabela 1).

Tabela 1. Caracterização das publicações científicas relacionadas à educação em saúde na formação de discentes de enfermagem, no período de 2012 a 2016. Jequié, Bahia, Brasil. 2017

\begin{tabular}{|c|c|c|c|c|}
\hline Autores & $\begin{array}{c}\text { Ano de } \\
\text { publicação }\end{array}$ & Objetivo & Tipo de Estudo & $\begin{array}{l}\text { Revista de } \\
\text { Publicação }\end{array}$ \\
\hline $\begin{array}{l}\text { Pinheiro SJ, Lucas } \\
\text { FEQ, Barreto LF, } \\
\text { Cordeiro MR, Cruz } \\
\text { M, Pereira FGF, et } \\
\text { al }^{11}\end{array}$ & 2016 & $\begin{array}{l}\text { Compreender as práticas de educação em } \\
\text { saúde no contexto da formação em } \\
\text { enfermagem. }\end{array}$ & $\begin{array}{l}\text { Estudo exploratório- } \\
\text { descritivo. }\end{array}$ & $\begin{array}{l}\text { Revista da Rede } \\
\text { de Enfermagem } \\
\text { do Nordeste. }\end{array}$ \\
\hline $\begin{array}{lr}\text { Holanda } & \text { VR, } \\
\text { Pinheiro } & \text { AKB } \\
\text { Holanda } & \text { ER, } \\
\underline{M C L}^{12} & \text { Santos }\end{array}$ & 2015 & $\begin{array}{l}\text { Avaliar uma hipermídia como estratégia } \\
\text { de ensino, a aprendizagem em ambiente } \\
\text { virtual e a atitude de acadêmicos de } \\
\text { Enfermagem para o ensino online das } \\
\text { doenças sexualmente transmissíveis. }\end{array}$ & $\begin{array}{l}\text { Estudo quase } \\
\text { experimental, do tipo } \\
\text { antes e depois. }\end{array}$ & $\begin{array}{l}\text { Revista Mineira } \\
\text { de } \\
\text { Enfermagem. }\end{array}$ \\
\hline $\begin{array}{l}\text { Lucchese R, Calixto } \\
\text { BS, Vera I, Paula NI, } \\
\text { Veronesi CL, } \\
\text { Fernandes } \mathrm{CNS}^{13}\end{array}$ & 2015 & $\begin{array}{l}\text { Analisar uma proposta didático- } \\
\text { pedagógica de ensino de tecnologias de } \\
\text { grupos, segundo o referencial teórico de } \\
\text { Pichón-Rivière, aplicado a graduandos em } \\
\text { Enfermagem. }\end{array}$ & $\begin{array}{l}\text { Estudo exploratório- } \\
\text { descritivo. }\end{array}$ & $\begin{array}{l}\text { Escola Anna } \\
\text { Nery Revista de } \\
\text { Enfermagem. }\end{array}$ \\
\hline $\begin{array}{l}\text { Oliveira MR, Leonel } \\
\text { ARA, Montezeli JH, } \\
\text { Gastaldi AB, Martins } \\
\text { EAP, Caveião C } 14\end{array}$ & 2015 & $\begin{array}{l}\text { Apresentar a concepção de graduandos } \\
\text { de enfermagem participantes de um } \\
\text { projeto integrado acerca da educação em } \\
\text { saúde sobre primeiros socorros. }\end{array}$ & $\begin{array}{l}\text { Estudo exploratório- } \\
\text { descritivo. }\end{array}$ & $\begin{array}{l}\text { Revista da Rede } \\
\text { de Enfermagem } \\
\text { do Nordeste. }\end{array}$ \\
\hline $\begin{array}{l}\text { Azevedo IC, Vale } L D, \\
\text { Araújo } \quad M G, \\
\text { Cassiano AN, Silva } \\
\text { HS, Cavalcante } R D^{15}\end{array}$ & 2014 & $\begin{array}{l}\text { Relatar a experiência vivenciada durante } \\
\text { as atividades de intervenção realizadas } \\
\text { com adolescentes e jovens de uma } \\
\text { instituição pública de ensino. }\end{array}$ & Relato de experiência. & $\begin{array}{l}\text { Revista de } \\
\text { Enfermagem do } \\
\text { Centro- } \\
\text { Mineiro. }\end{array}$ \\
\hline $\begin{array}{l}\text { JESUS, I.S.; SENA, E. } \\
\text { L. S.; ANDRADE, } \\
\text { L.M }^{16}\end{array}$ & 2014 & $\begin{array}{l}\text { Descrever a percepção de docentes e } \\
\text { graduandos em enfermagem sobre a } \\
\text { experiência dialógica nos espaços } \\
\text { informais e sua relação com a formação } \\
\text { em saúde. }\end{array}$ & $\begin{array}{l}\text { Estudo exploratório- } \\
\text { descritivo. }\end{array}$ & $\begin{array}{l}\text { Revista Latino- } \\
\text { Americana de } \\
\text { Enfermagem. }\end{array}$ \\
\hline $\begin{array}{lr}\text { Valença } & \mathrm{CN}, \\
\text { Germano } & \mathrm{RM}, \\
\text { Malveira } & \mathrm{FAS}, \\
\text { Azevêdo } & \text { LMN, } \\
\text { Oliveira AG }{ }^{17} & \\
\end{array}$ & 2014 & $\begin{array}{l}\text { Identificar as contribuições do Curso de } \\
\text { Extensão Vivências e Estágios na } \\
\text { Realidade do Sistema Único de Saúde } \\
\text { (SUS), no contexto da saúde coletiva para } \\
\text { a formação em saúde/enfermagem. }\end{array}$ & $\begin{array}{l}\text { Estudo exploratório- } \\
\text { descritivo. }\end{array}$ & $\begin{array}{l}\text { Revista } \\
\text { Enfermagem } \\
\text { UERJ. }\end{array}$ \\
\hline 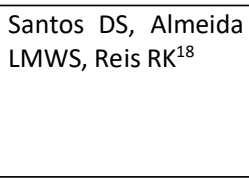 & 2013 & $\begin{array}{l}\text { Relatar a experiência de criação e } \\
\text { desenvolvimento do PET-Saúde } \\
\text { Enfermagem e seu papel de mudança na } \\
\text { formação de Enfermagem e no } \\
\text { fortalecimento da ESF em Maceió. }\end{array}$ & Relato de experiência. & $\begin{array}{ll}\text { Revista da } \\
\text { Escola de } \\
\text { Enfermagem da } \\
\text { USP. }\end{array}$ \\
\hline $\begin{array}{lr}\text { COLOMÉ, J. } & \text { S.; } \\
\text { OLIVEIRA, D. L. L. C } & C^{19}\end{array}$ & 2012 & $\begin{array}{l}\text { Analisar as concepções de estudantes de } \\
\text { enfermagem acerca da educação em } \\
\text { saúde e quais são seus sujeitos e agentes. }\end{array}$ & $\begin{array}{l}\text { Estudo exploratório- } \\
\text { descritivo }\end{array}$ & $\begin{array}{l}\text { Revista Texto \& } \\
\text { Contexto } \\
\text { Enfermagem. }\end{array}$ \\
\hline $\begin{array}{lr}\text { SANTOS, } & \text { C.M.; } \\
\text { OLIVEIRA, S. M. G } \text { G }^{20}\end{array}$ & 2012 & $\begin{array}{l}\text { Relatar a experiência como bolsista, a fim } \\
\text { de estimular outros graduandos a se } \\
\text { envolverem em atividades semelhantes e } \\
\text { também produzirem outros estudos na } \\
\text { área. }\end{array}$ & Relato de experiência. & $\begin{array}{l}\text { Revista Baiana } \\
\text { de } \\
\text { Enfermagem. }\end{array}$ \\
\hline
\end{tabular}

Fonte: Dados da pesquisa. 
Assim, para a discussão desta pesquisa, foi aplicado o critério de análise dos tópicos que proporcionou categorizar, elucidar e classificar os dados. Desse agrupamento mostra-se duas categorias: metodologias para a formação em educação em saúde e concepções de discentes de enfermagem sobre educação em saúde na formação.

\section{Metodologias para a formação em educação em saúde}

Entre as metodologias em educação em saúde pode-se utilizar o ambiente virtual. Sendo esta estratégia motivadora ao discente de enfermagem que adquire ainda mais conhecimentos com $o$ acesso às atualizações tecnológicas, além de complementar o ensino da sala de aula. A aprendizagem online relaciona-se também com a possibilidade de utilização de recursos audiovisuais, aplicativos em ações educativas a serem planejadas na atuação como enfermeiro ${ }^{12}$.

Uma oportunidade para o discente de enfermagem no estudo através da metodologia da problematização na educação em saúde é o PET-Saúde enfermagem. Este projeto possibilita o desenvolvimento de ações de educação em saúde como atividade base e prioritários com os discentes e usuários e família. Estudo destaca a relevância do trabalho realizado neste projeto por respeitar as particularidades do curso de enfermagem e da profissão através de ações de dimensões: "educação em saúde e metodologia do processo de trabalho de Enfermagem na ESF". De forma que as ações podem proporcionar o enriquecimento da formação desses discentes por meio da pesquisa-ação em educação em saúde na Atenção Básica ${ }^{21}$.

A metodologia de trabalho em enfermagem através de tecnologias de grupo pode extrapolar o modelo de ensino tradicional, de forma a ser vista como uma ferramenta para a aprendizagem e a realização de educação em saúde. Sendo que o grupo estimula à capacidade de enfrentamento de situações-problema, elaboração de atividades em coletivo, estudo e compartilhamento de aprendizado com outros indivíduos e a valorização da comunicação em saúde, a escuta, o cuidado ${ }^{13}$.

O processo educativo problematizador oportunizado na formação do discente de enfermagem também através do PET-Saúde direciona o discente a valorizar as necessidades de saúde da comunidade, além de integrar o ensino, a pesquisa e a extensão. E nesse processo de ensino-aprendizagem também em trabalhos grupais com demais discentes, além do envolvimento do docente (preceptor) e de profissionais da equipe de Saúde da Família, o discente vivencia desde o planejamento (com levantamento de problemas para o estabelecimento de prioridades nas ações de educação em saúde), assim como na intervenção (com metodologias ativas) e avaliação das práticas educativas ${ }^{21}$.

As contribuições do Projeto de Extensão Vivências e Estágios na Realidade do SUS (VERSUS) também é uma estratégia para despertar no discente de enfermagem seu protagonismo para uma atuação voltada para a integralidade, também na realização de educação em saúde com metodologias ativas e na articulação entre ensino, serviço e extensão ${ }^{17}$.

A educação em saúde vivenciada em estágio supervisionado por sua vez, permite a formação de discentes de enfermagem além das ações a serem realizadas na USF, como ambientes: escola, igreja, comunidade. Dessa forma, o estágio supervisionado é um momento educacional percebido como espaço de interação entre os discentes, equipe de Saúde da Família, usuários e família, seja no cenário da unidade de saúde, como nos equipamentos sociais ${ }^{15}$.

Assim como o estágio extracurricular também é uma metodologia percebida como possibilidade para aquisição de conhecimentos nos quais busca-se aprofundamento, além da realização de educação em saúde em um outro cenário de saúde. O discente realiza além da observação, a intervenção em assistência, educação para uma experiência integral. Dessa forma, o discente ativo solicitará na universidade o diálogo entre instituições para a aquisição de autorização formal para tais vivências e agregará esse aprendizado quando estiver em exercício profissional $^{20}$

Em estudo sobre estágio supervisionado em enfermagem realizado em instituição de ensino médio com abordagem de temas direcionados pelos adolescentes percebe-se que o discente de enfermagem tende a articular os saberes adquiridos em saúde e consolidados no cotidiano com os atores envolvidos. Nesta vivência, ao ser realizado o levantamento de necessidades de saúde dos alunos foi percebida a falta de ações da equipe de Saúde da Família nesse cenário escolar. Portanto, a relevância do trabalho de educação em saúde em instituições de ensino durante a formação em enfermagem é necessária por constituir um vínculo interinstitucional e a possibilidade de 
compartilhamento de experiências, aprendizados. Além de proporcionar a aproximação de ações de saúde com o setor da educação, diante de possíveis ações insuficientes pela equipe de Saúde da Família. Assim, os futuros enfermeiros compreenderão esse espaço (a escola) também como de sua responsabilidade de atuação ${ }^{15}$.

A educação em saúde na formação de discentes de enfermagem pode ser percebida como estratégia para a inserção e a atuação efetiva na ESF. Através de metodologias ativas e problematizadoras de educação rompe-se com a formação em saúde no modelo tradicional, pois há um fazer crítico e reflexivo, em substituição às práticas pontuais e lineares.

Acredita-se que por meio de reflexões sobre as metodologias e estratégias na formação de educação em saúde haverá o fortalecimento da transversalidade do processo ensinoaprendizagem de educação em saúde nos cursos de graduação em Enfermagem, assim como permite a possibilidade de suscitar um novo olhar dos gestores nas instituições educacionais de ensino superior de Enfermagem a respeito dessa temática, fornecendo-lhes subsídios para refletir sobre a organização da formação oferecida aos discentes.

Concepções de discentes de enfermagem sobre educação em saúde na formação

Em estudo sobre a concepção de discentes de enfermagem sobre a educação em saúde verificou-se a predominância de conceituação e percepção diante do saber biomédico. Considerando que os discentes acreditam na transmissão de informações científicas, na prescrição de mudanças de hábitos e comportamento para os usuários, em abordagem em sua maioria a serem centradas na doença. Sendo mencionado que nas experiências curriculares vivenciadas foram exigidas uma atuação com ênfase nos saberes técnicocientíficos ${ }^{19}$.

A apresentação de espaços informais (grupos sociais) para a possibilidade de diálogo entre os envolvidos e a realização de atividades que extrapolam o planejamento de ações curriculares devem ser valorizados. Sendo exigida aos discentes de enfermagem a formação mais ampla, com agregação das competências pedagógicas às competências técnicas ${ }^{16}$.

A concepção de discentes de enfermagem sobre a participação em projeto de educação em saúde com a temática de primeiros socorros é percebida como uma ferramenta para o aprimoramento do conhecimento dessa temática.
Além de permitir que o futuro enfermeiro possua conhecimento de aspectos teóricos e práticos com estratégias, possíveis metodologias a serem utilizadas em ações em grupo, necessidade da adaptação da linguagem dependendo do público com o qual se está trabalhando e a importância de intensificar o empoderamento do usuário, assim como do discente ${ }^{14}$.

Os discentes de enfermagem demonstram compreensão sobre a importância da educação em saúde na formação, sendo necessário destacar que tal formação deve ser realizada através de aulas teóricas e práticas. Sendo que nesta prática precisa-se respeitar o nível cultural dos indivíduos, sendo esse momento fonte de aprendizado para o discente que aprende a cuidar do indivíduo e do coletivo, assim como a comunicar-se com criatividade nos níveis primário, secundário e terciário de atenção à saúde ${ }^{11}$.

As Diretrizes Curriculares Nacionais (DCN) por sua vez demonstram no Art. 50 que a formação do Enfermeiro deve atender as necessidades sociais de saúde, com ênfase no Sistema Único de Saúde (SUS) e é necessário que os discentes sejam dotados de conhecimentos para o exercício de competências e habilidades, entre elas: planejar e implementar ações de educação em saúde considerando a singularidade dos grupos sociais e do modo de vida. No Art. 6 o entre os conteúdos essenciais na formação do Enfermeiro destaca-se a capacitação pedagógica e deve estar relacionado com o sujeito, família, comunidade para a integralidade das ações do cuidar. Assim como o Art. 9o no qual é mencionado que o Curso de Graduação em Enfermagem deve ter um projeto pedagógico construído para o discente como sujeito de aprendizagem $^{9}$.

Revela-se a necessária responsabilidade das universidades na formação de enfermeiros críticos e que sejam comprometidos com a realidade social, política, cultural para o fortalecimento do SUS também por meio de ações educativas ${ }^{19}$. Pois, as transformações sociais e econômicas vêm exigindo necessárias mudanças na formação de futuros profissionais da saúde o que exige a discussão frequente nas instituições de ensino formadoras desses profissionais e a transversalidade na formação com a abordagem além da técnica, mas a coresponsabilização por indivíduos envolvidos com suas ações, exercício da cidadania e do cuidado ao outro ${ }^{22}$.

A formação do discente deve se relacionar a uma formação crítica, reflexiva, na qual sejam presentes os questionamentos, as investigações, 
as problematizações. Ao discente, de acordo a sua história, singularidades já construídas antes mesmo do período de formação, se percebe e é sensibilizado para se perceber constituinte do processo de descobertas e conhecedor das estratégias e ferramentas apresentadas pelos docentes ${ }^{23}$.

O processo de ensino-aprendizagem mais efetivo na formação de futuros enfermeiros deve ser valorizado para a consolidação do Sistema Único de Saúde e para a melhoria da atuação desses profissionais. A serem profissionais mais criativos e conscientes da relevância do conhecimento popular e adequação das ações educativas diante da realidade encontrada em cada comunidade, assim como pela singularidade do indivíduo, família.

\section{Conclusões}

Os trabalhos científicos analisados possibilitaram verificar as características da formação do enfermeiro em educação em saúde comprometendo-se com as demandas do SUS e que a integração ensino-serviço se configura como estratégia para superar a dicotomia entre ensino e prática.

Contudo ressalta-se a necessidade de estrutura organizacional dos cursos de enfermagem para oportunizar a participação dos discentes de enfermagem em projetos de extensão, vínculos com demais instituições educacionais para o intercâmbio de experiências e aprendizado.

A formação do enfermeiro deve ser crítica e reflexiva, tendo o cuidado como eixo norteador, de modo a estimular que o acadêmico de enfermagem seja comprometido com a atuação criativa, reflexiva e humanizada.

Dentre os achados também ficou perceptível a necessidade do pensamento crítico ser desenvolvido durante a graduação em enfermagem, demandando ensino criativo e flexível. De forma que o discente compreenda sua inserção no cenário acadêmico e social enquanto sujeito ativo da sua formação e detentor de perfil profissional comprometido com a profissão e transformação social.

\section{Referências}

1. Lopes R, Tocantins FR. Health Promotion and Critical Education. Interface - Comunic., Saude, Educ. 2012; 16 (40): 235-48.
2. Pinafo E, Nunes EFPA, González AD. A educação em saúde na relação usuáriotrabalhador no cotidiano de equipe de saúde da família. Cien Saúde Colet. 2012; 17(7): 1825-32.

3. Carneiro ACLL, Souza V, Godinho LK, Faria ICM, Silva KL, Gazzinelli MF. Educação para a promoção da saúde no contexto da atenção primária. Rev Panam Salud Publica. 2012; 31(2):115-20.

4. Fonseca GS, Paulino TSC, Morais IF, Valença CN, Germano RM. Percepção de usuários e profissionais de saúde sobre o Sistema Único de Saúde no município de Santa Cruz-RN. Rev Bras Promoç Saúde. 2012; 25 (4): 455-61.

5. Figueiredo MFS, Rodrigues Neto JF, Leite MTS. Educação em saúde no contexto da Saúde da Família na perspectiva do usuário. Interface Comunic., Saude, Educ. 2012; 16 (41): 315-29.

6. Gazzinelli MF, Souza V, Fonseca RMGS, Fernandes MM, Carneiro ACLL, Godinho LK.Educational group practices in primary care: interaction between professionals, users and knowledge.Rev Esc Enferm USP. 2015; 49(2): 282-9.

7. Brasil. Ministério da Saúde. Política Nacional de Atenção Básica. Departamento de Atenção Básica. Brasília: Ministério da Saúde, 2017.

8. Silva AMB, Wysock AD. Educação em saúde e grupos educativos: abordagens importantes. In: Santos AS, Paschoal VD. (Orgs.). Educação em saúde e enfermagem. Barueri, São Paulo: Manole; 2017. p. 73-94.

9. Brasil. Conselho Nacional de Educação. Câmara de Educação Superior. Parecer CNE/CES no 1133, de 7 de agosto de 2001. Dispõe sobre as Diretrizes Curriculares de Medicina, Enfermagem e Nutrição. Brasília: Distrito Federal; 2001.

10. Bardin L. Análise de conteúdo. São Paulo: Edições 70, 2011.

11. Pinheiro SJ, Lucas FEQ, Barreto LF, Cordeiro MR, Cruz M, Pereira FGF, Barbosa AL. Conceptions of health education practices in the context of Nursing Education. Rev Rene. 2016; 17(4): 545-52.

12. Holanda VR, Pinheiro AKB, Holanda ER, Santos MCL. Ensino e aprendizagem em ambiente virtual: atitude de acadêmicos de enfermagem. Rev Min Enferm. 2015; 19 (1):14147.

13. Lucchese R, Calixto BS, Vera I, Paula NI, Veronesi CL, Fernandes CNS. O ensino de práticas grupais em enfermagem norteado pelo referencial de Pichon-Rivière. Esc Anna Nery. 2015; 19(2): 212-19. 
14. Oliveira MR, Leonel ARA, Montezeli JH, Gastaldi AB, Martins EAP, Caveião C. Conception of under graduate nursing students on the practice of health education on first aid. Rev Rene. 2015; 16(2): 150-8.

15. Azevedo IC, Vale $L D$, Araújo $M G$, Cassiano AN, Silva HS, Cavalcante RD. Compartilhando saberes através da educação em saúde na escola: interfaces do estágio supervisionado em enfermagem. R. Enferm. Cent. O. Min. 2014; 4(1): 1048-56.

16. Jesus IS, Sena ELS, Andrade LM. Aprendizagem nos espaços informais $e$ ressignificação da existência de graduandos de enfermagem. Rev. Latino-Am. Enfermagem. 2014; 22(5): 731-8.

17. Valença CN, Germano RM, Malveira FAS, Azevêdo LMN, Oliveira AG. Articulação teoria/prática na formação em saúde e a realidade do Sistema Único de Saúde. Rev enferm UERJ. 2014; 22(6): 830-5.

18. Santos DS, Almeida LMWS, Reis RK. Programa de Educação pelo Trabalho para Saúde: experiência de transformação do ensino e prática de enfermagem. Rev Esc Enferm USP. 2013; 47(6): 1431-6.

19. Colomé JS, Oliveira, DLLC. Educação em saúde: por quem e para quem? A visão de estudantes de graduação em enfermagem.Texto Contexto Enferm. 2012; 21(1): 177-84.

20. Santos CM, Oliveira, SMG. Estágio extracurricular como complemento das práticas em saúde na graduação. Revista Baiana de Enfermagem. 2012; 26 (2):541-46.

21. Santos DS, Almeida LMWS, Reis RK. Programa de Educação pelo Trabalho para Saúde: experiência de transformação do ensino e prática de enfermagem. Rev Esc Enferm USP. 2013; 47(6):1431-6.

22. Fontoura EG, La Torre MPS, Rosa DOS, Vieira TT. Processo de formação da enfermeira para um agir ético. Revista Baiana de Enfermagem. 2011; 25(1): 59-68.

23. Fernandes MFP, Vaz DR. Processo ensino/aprendizagem-ensinagem. In: Prado C (Org.). Práticas pedagógicas em Enfermagem: processo de reconstrução permanente. 1. ed. São Caetano do Sul, SP: Difusão Editora, 2013. p.6374.

\section{Endereço para Correspondência}

Universidade Estadual do Sudoeste da Bahia UESB

Rua, Av. José Moreira Sobrinho, s/n - Jequiezinho, Jequié - BA

CEP.: 45206-190

e-mail: tatiana_almeidacouto@hotmail.com

Recebido em 23/04/2018

Aprovado em 17/07/2018

Publicado em 20/12/2018 